\title{
PERICARDITE COMO MANIFESTAÇÃO CLÍNICA EM PACIENTE COM DOENÇA DE CROHN: RELATO DE CASO
}

\author{
GUIRALDELLO, Lidiane ${ }^{1}$ \\ CERRUTI, Cássio Henrique ${ }^{1}$ \\ CEARÁ, Christiane Damasceno Arcelino ${ }^{2}$ \\ CUNHA JUNIOR, Wilson ${ }^{3}$
}

ISSUE DOI: $10.3738 / 1982.2278 .3550$

\begin{abstract}
RESUMO: Introdução: A doença de Crohn (DC) é uma patologia de etiologia incerta e se apresenta com inflamação transmural do trato gastrintestinal. É aventada a hipótese de que uma desregulação do sistema imunológico seria a base fisiopatológica da doença, tendo uma origem multifatorial envolvendo genética e fatores ambientais. A moléstia transcorre com sinais e sintomas muitas vezes inespecíficos, podendo envolver basicamente todos os sistemas, o que pode retardar o diagnóstico. É característico da enfermidade, manifestações extraintestinais, das quais a artropatia de grandes articulações é a mais prevalente, sendo cardíacas raras, com poucos casos de pericardite relatados na literatura. Relato de caso: Paciente, masculino, 32 anos, com quadro diarreico há um mês, resolvido espontaneamente e associado à perda ponderal de $15 \mathrm{~kg}$. Procurou atendimento médico devido a dor torácica pleurítica, com supradesnivelamento do segmento ST ao eletrocardiograma e enzimas cardíacas normais. Evoluiu ao longo da internação com novo quadro diarreico associado a hematoquezia, além de úlceras orais e uveíte. Realizado colonoscopia, com resultado de colite inflamatória característico de DC. Iniciado pulsoterapia com metilprednisona e antiboticoterapia. Paciente teve remissão do quadro agudo e recebeu alta com seguimento ambulatorial. Conclusão: Diversas manifestações clínicas podem estar presentes em pacientes com DC, sendo a pericardite considerada como rara, e embora geralmente relacionada aos efeitos colaterais das medicações utilizadas no tratamento da DC, neste caso, cursou como manifestação primária da doença. Com este caso, espera-se alertar os profissionais quanto ao diagnostico precoce e sinalizar quanto a associação de DC e pericardite.
\end{abstract}

Palavras chave: Trato gastrointestinal, Inflamação, Pericárdio.

\section{PERICARDITE AS CLINICAL MANIFESTATION IN PATIENT WITH CROHN'S DISEASE: CASE REPORT}

SUMMARY: Introduction: Crohn's disease (CD) is a pathology of uncertain etiology and presents with transmural inflammation of the gastrointestinal tract. It is hypothesized that a dysregulation of the immune system would be the pathophysiological basis of the disease, having a multifactorial origin involving genetics and environmental factors. The disease occurs with signs and symptoms that are often nonspecific, and may involve basically all systems, which can delay the diagnosis. It is characteristic of the disease, extraintestinal Introduction: Crohn's disease (CD) is a pathology of uncertain etiology and presents with transmural inflammation of the gastrointestinal tract. It is hypothesized that a dysregulation of the immune system would be the pathophysiological basis of the disease, having a multifactorial origin involving genetics and environmental factors. The disease occurs with signs and symptoms that are often nonspecific, and may involve basically all systems, which can delay the diagnosis. It is characteristic of the disease, extraintestinal manifestations, of which arthropathy of large joints is the most prevalent, being rare cardiac, with few cases of pericarditis reported in the literature. Case report: Patient, male, 32 years old, with diarrhea for one month, resolved spontaneously and associated with weight loss of $15 \mathrm{~kg}$. He sought medical attention due to pleuritic chest pain, with ST segment elevation to the electrocardiogram and normal cardiac enzymes. It evolved during hospitalization with a new diarrheal disease associated with hematochezia, in addition to oral ulcers and uveitis. A colonoscopy was performed, resulting in inflammatory colitis characteristic of CD. Initiated pulse therapy with methylprednisone antibotic therapy. Patient had remission of acute and was discharged with outpatient follow-up. Conclusion: Several clinical manifestations may be present in patients with $\mathrm{CD}$, and pericarditis is considered to be rare, and although generally related to the side effects of medications used in the treatment of $\mathrm{CD}$, in this case, it was the primary manifestation of the disease. With this case, it is expected to alert the professionals about the early diagnosis and to indicate the association of CD and pericarditis.

\footnotetext{
${ }^{1}$ Universidade de Franca - Estudante de Medicina

${ }^{2}$ Santa Casa de Misericórdia de Franca - Médica

${ }^{3}$ Universidade de Franca - Médico e Docente do Curso de Medicina
} 
Keywords: Gastrointestinal tract, Inflammation, Pericardium.

\section{INTRODUÇÃO}

A Doença de Crohn é uma patologia de etiologia incerta que se apresenta com uma inflamação transmural do trato gastrintestinal. É aventada a hipótese de que uma desregulação do sistema imunológico seria a base da fisiopatologia da doença, tendo, no geral, as doenças intestinais inflamatórias, uma origem multifatorial que engloba genética e fatores ambientais. Os sintomas podem se estender por todo o trato gastrointestinal, afetando desde a cavidade oral até a região perianal. (PEPPERCOM, 2018; DOGANAY, 2006)

Estima-se que $80 \%$ dos pacientes apresentam a doença envolvendo íleo distal, sendo acometidos exclusivamente por ileíte, enquanto aproximadamente 50\% manifestam-se com ileocolite. Já $20 \%$ dos casos, são identificados com doença limitada ao cólon. (PEPPERCOM, 2018)

A moléstia transcorre com sinais e sintomas muitas vezes inespecíficos de doença inflamatória intestinal, podendo envolver basicamente todos os sistemas, o que retarda o diagnóstico da patologia. Dentre as manifestações clínicas as mais prevalentes são fadiga, febre, diarreia crônica com ou sem sangramento, dor abdominal e perda de peso. Doença perianal pode aparecer no curso da afecção, assim como síndromes desabsortivas, que são causadas pela inflamação da mucosa do trato gastrointestinal, responsáveis pelo comprometimento do estado geral do indivíduo. (DOGANAY, 2006)

É característico de a enfermidade apresentar manifestações extraintestinais, as quais geralmente estão relacionadas com a atividade da doença. Destas a mais comum é a artropatia que envolve grandes articulações e está presente em aproximadamente 20\% dos pacientes. Podem ocorrer também, em cerca de $10 \%$ dos enfermos, distúrbios de pele que incluem mais comumente o eritema nodoso e o pioderma gangrenoso. Manifestações do sistema ocular como uveíte, irite e epsclerite, também podem estar presentes. São menos frequentes sintomas hematológicos (tromboembolismo), renais (glomerulonefrite, nefropatia), neurológicos (esclerose múltipla), pulmonares (bronquiectasia, asma, bronquiolite obliterante) e cardíacos (pericardite). As manifestações cardíacas da Doença de Crohn são raras sendo que há pouco mais de uma centena de casos de pericardite relatados na literatura associada à moléstia, dado que o diagnóstico da sua associação foi realizado por exclusão de outras etiologias.( PEPPERCOM, 2018; DOGANAY, 2006; GARCIA-MORA, 2006)

\section{RELATO DE CASO}

\section{Número de protocolo CAAE: 99733718000005438.}

Paciente masculino, 32 anos, previamente hígido, admitido com quadro de dispneia e dor torácica intermitente, ventilatório dependente, com piora em decúbito, associada á palpitação. Paciente referiu que há aproximadamente um mês tinha apresentado episódios de diarreia, com resolução espontânea, além de perda ponderal involuntária de aproximadamente $15 \mathrm{~kg}$, adinamia e hiporexia.

Ao exame físico, manteve-se eupneico e com murmúrio vesicular diminuído em ambas as bases pulmonares. Na ausculta cardíaca havia sopro sistólico ++/6+ em foco mitral. Demais órgãos e aparelhos examinados não mostraram alterações ao exame no momento da internação.

Foram realizados exames complementares, sendo que o hemograma demonstrou anemia microcítica e hipocrômica; a enzimas cardíacas estavam dentro dos parâmetros aceitos e FAN + 1/160 nuclear pontilhado grosso. Além dos exames laboratoriais, foi solicitado um etrocardiograma, o qual 
demonstrou supradesnivelamento de segmento ST em parede anterior, lateral e inferior do miocárdio. Decidiu-se então por realizar um ecocardiograma no qual foi possível encontrar dilatação moderada do átrio esquerdo, desempenho sistólico e mobilidade segmentar biventricular preservadas. No ventrículo esquerdo havia hipertrofia miocárdica $20 \mathrm{~mm}$ em região látero- apical e fração de ejeção de $60 \%$.

Durante a internação, o paciente evoluiu com novos episódios diarreicos com presença de enterorragia. Alem disso, iniciou com úlceras orais dolorosas, hiperemia conjuntival à esquerda e linfonodomegalia cervical e inguinal (com linfonodos menores que um centímetro e consistência fibroelástica). Em virtude disso, optou-se por realizar uma colonoscopia, na qual se observou múltiplas úlceras em mucosa anal e em todos os segmentos colônicos, intercaladas por pequenas ilhas de mucosa normal e alguns segmentos com mucosa em pedra de calçamento, íleo terminal normal. Achados compatíveis com pancolite inflamatória, sugestiva de Doença de Crohn.

Decidiu-se, então, iniciar tratamento com pulsoterapia com metilprednisona durante três dias e antibioticoterapia por sete dias, havendo boa resposta clínica e estabilização do quadro clínico do paciente.

\section{DISCUSSÃO}

De acordo com o caso apresentado, o paciente em questão iniciou quadro clínico compatível ao de uma Doença Inflamatória Intestinal, porém só buscou assistência médica quando deparado com sintomas precordiais, compatíveis com pericardite, os quais foram confirmados posteriormente.

Quanto ás manifestações clínicas da Doença de Crohn, embora muito variáveis, podem se apresentar inicialmente com diarreia prolongada ou crônica, com ou sem sangramento, dor abdominal, fadiga, perda ponderal e febre. A diarreia é uma das apresentações mais comum, sendo flutuante em muitos casos, o que pode retardar a busca por assistência médica, como ocorrido no caso relatado. Contudo, essas manifestações podem ser concomitante a outros sintomas característicos da patologia como desnutrição, palidez cutaneomucosa, massa abdominal, distensão ou fistulização na parede abdominal, alem de fissuras anais. Já as manifestações extraintestinais estão diretamente relacionadas com a intensidade da atividade inflamatória intestinal, ocorrendo de forma mais rotineira as apresentações cutâneas, articulares e oculares. (BURGMANN, 2006; PEPPERCORN, 2018; GAMA, 2011)

De forma geral, a doença de Crohn pode ser diagnosticada por meio de dados clínicos, sendo anamnese, exame físico e proctológico essenciais, assim como, com exames complementares que incluem dados laboratoriais, radiológicos, endoscópicos e histológicos. No caso em questão, além da forma clínica, pode ser observado que o diagnóstico foi confirmado com a realização da colonoscopia a qual demonstrou uma mucosa acometida por lesões ulcerosas em padrão "paralelepípedo" (cobblestone), altamente sugestivo da patologia. (GAMA, 2011)

Já em relação aos sintomas torácicos, foi aventada a hipótese de pericardite em virtude desta se apresentar muitas vezes com dor torácica associada á dispneia, sintomas que podem estar relacionados ao debito cardíaco diminuído em resposta a um esforço, além das alterações de eletrocardiogramas que sugerem tal afecção como o supradesnivelamento difuso do segmento ST. A elevação do seguimento ST, comum da isquemia do infarto agudo do miocárdio, neste caso representa uma atividade inflamatória subepicárdica do miocárdio adjacente ao pericárdio. Outras alterações que poderiam estar presentes seria o infradesnivelamento do segmento PR e taquicardia sinusal. (FRIEDMANN, 2016; HOIT, 2018)

Como já mencionado, raros são os casos registrados de manifestações extraintestinais cardíacas na doença inflamatória intestinal. Contudo essa relação continua incerta, uma vez que muitos casos reportados estiveram ligados com o uso da mesalazina, sulfasalazina e azatioprina fármacos utilizados no tratamento da patologia. Nestes casos, associação das drogas com o acometimento do pericárdio é 
explicado por uma reação de hipersensibilidade aguda, desencadeada no início da terapêutica. Este fato, porém, não coincide com o caso do presente estudo, uma vez que as manifestações de pericardite surgiram antes do inicio do tratamento do paciente em questão. (DOGANAY, 2006; GARCIA, 2006)

A relação estabelecida entre a pericardite e a medicação pode ser difícil devido aos diversos fatores que tem a possibilidade de provocar pericardite como infecções virais e bacterianas. No caso descrito, os diagnósticos diferenciais principais foram excluídos, chegando-se à relação entre as patologias.(KIYOMATSU, 2015)

A mensuração da atividade da doença pode ser feita por meio do Índice de Atividade da Doença de Crohn que leva em consideração o número de evacuações líquidas, dor abdominal, sensação de bem estar do paciente, número de complicações, necessidade ou não do uso de antidiarreico, presença de massas abdominais e a dosagem de hematócrito. As drogas de eleição disponíveis em nosso meio são aminossalicilato (mesalazina, sulfassalazina), antibiótico para flora característica intestinal (anaeróbios e Gram negativos), corticoide (pulsoterapia com metilprednisona), imunossupressores (azatioprina, metotrexato) ou ainda a terapia biológica (infliximabe). Aplica-se o esquema step up sendo a cirurgia com ressecção de fragmentos intestinais acometidos a última opção. No caso descrito, o controle dos sintomas associados foi alcançado fazendo-se uso das medicações iniciais. Embora haja um numero reduzido de casos de pericardite relacionada à Doença de Crohn, é indiscutível que o tratamento evita complicações e exacerbações da patologia. (GAMA, 2011.)

\section{CONCLUSÃO}

Diversas manifestações clínicas e extraintestinais podem estar presentes em pacientes com Doença de Crohn, sendo a pericardite considerada rara na literatura. Além disso, em alguns casos descritos, as manifestações cardíacas geralmente estão relacionada aos efeitos colaterais das medicações utilizadas no tratamento da patologia. Neste caso, porém foi possível questionar a hipótese de que a pericardite tenha sido uma das manifestações extratintestinais primárias da doença, uma vez que os sintomas estavam presentes antes do diagnostico e tratamento da doença de base. Com isso, espera-se informar e sinalizar aos profissionais da saúde quanto a associação da Doença de Crohn com a pericardite, além de orientar sobre a importância do diagnostico e tratamento precoce da doença, afim de se ter melhor controle dos sintomas e das complicações que podem acometer o paciente.

\section{REFERÊNCIAS}

BURGMANN, T et al. The manitoba inflammatory bowel disease cohort study: prolonged symptoms before diagnosis--how much is irritable bowel syndrome? Clin Gastroenterol Hepatol., [S. l.], 2006. Disponível em: https://www.ncbi.nlm.nih.gov/pubmed/16630762. Acesso em: 5 jan. 2019.

DOGANAY, L et al. Mesalazine-induced myopericarditis in a patient with ulcerative colitis. International Journal of Colorectal Disease, [S. l.], março 2006. Disponível em: https://link.springer.com/article/10.1007\%2Fs00384-004-0706-1?LI=true. Acesso em: 4 jan. 2019

FRIEDMANN, A. A. Eletrocardiograma típico de pericardite. Diagn Tratamento, [S. l.], 2017. Disponível em: http://docs.bvsalud.org/biblioref/2017/08/848013/rdt_v22n3_119-120.pdf. Acesso em: 5 jan. 2019

GARCÍA-MORÁN , S et al. Myopericarditis and mitral insufficiency associated with ulcerative colitis treated with mesalazine. Inflamm Bowel Dis, [S. l.], p. 334-5, 12 abr. 2006. Disponível em:

https://www.ncbi.nlm.nih.gov/pubmed/16633055. Acesso em: 6 jan. 2019. 
GAMA , A. H. et al. Doença de Crohn intestinal: manejo. Revista da Associação Médica Brasileira, São Paulo, Jan/Fev 2011. Disponível em: http://www.scielo.br/scielo.php?script=sci_arttext\&pid=S010442302011000100006. Acesso em: 5 jan. 2019.

KIYOMATSU , H et al. Mesalazine-induced Pleuropericarditis in a Patient with Crohn's Disease. Internal medicine, Tokyo, Japão, p. 1605-8, 1 jul. 2015. Disponível em: https://www.ncbi.nlm.nih.gov/pubmed/26134190. Acesso em: 6 jan. 2019.

HOIT, B.D. Constrictive pericarditis. Uptodate, [S. l.], 2018. Disponível em: ttps://www.uptodate.com/contents/constrictive-pericarditis. Acesso em: 3 jan. 2019.

PEPPERCORN, M. A.; KANE, S. V. Clinical manifestations, diagnosis and prognosis of Crohn disease in adults. Uptodate, [S. l.], 2018. Disponível em: https://www.uptodate.com/contents/clinicalmanifestations-diagnosis-and-prognosis-of-crohn-disease-in-adults. Acesso em: 5 jan. 2019. 